\title{
Téoros
}

Revue de recherche en tourisme

\section{La Fédération des agricotours du Québec face au défi de l'agrotourisme}

\section{Anne Marchard}

Volume 20, numéro 2, été 2001

Tourisme rural

URI : https://id.erudit.org/iderudit/1071641ar

DOI : https://doi.org/10.7202/1071641ar

Aller au sommaire du numéro

Éditeur(s)

Université du Québec à Montréal

ISSN

0712-8657 (imprimé)

1923-2705 (numérique)

Découvrir la revue

Citer cet article

Marchard, A. (2001). La Fédération des agricotours du Québec face au défi de l'agrotourisme. Téoros, 20(2), 33-38. https://doi.org/10.7202/1071641ar d'utilisation que vous pouvez consulter en ligne.

https://apropos.erudit.org/fr/usagers/politique-dutilisation/ 


\section{La Fédération des agricotours du Québec face au défi de l'agrotourisme}

\section{Anne Marchard}

\section{Une prise de conscience}

C réée il y a déjà 26 ans, à l'initiative de l'Union des producteurs agricoles (UPA), avec l'aide du ministère de l'Agriculture, des Pêcheries et de l'Alimentation du Québec (MAPAQ), la Fédération des Agricotours du Québec (FAQ) est devenue le plus grand réseau de qualité de chambres d'hôtes et de petites auberges typiques du Québec. Aujourd'hui, on connait davantage la FAQ pour ses programmes d'hébergement, le « Gîte du Passant » et «l'Auberge du Passant », alors que son offre est née de programmes agrotouristiques, soit le « Gîte à la Ferme » et la « Promenade à la Ferme», auxquels se sont ajoutés, dans les années quatrevingts, la « Table Champêtre » et, tout récemment, le « Relais du Terroir ». L'objectif de la FAQ était de promouvoir et de démystifier la profession de producteur agricole par des activités touristiques complémentaires à la ferme. Ainsi, au début des années soixante-dix, une multitude de modestes services agrotouristiques se sont développés chez les agriculteurs, encouragés par l'arrivée de la FAQ. La structure et l'organisation de la FAQ avaient alors incité l'agrotourisme à prendre son envol.

Au tournant des années quatre-vingts, la spécialisation de l'agriculture a eu pour effet de ralentir le développement de l'agrotourisme au Québec. Par contre, ce tournant a véritablement marqué le développement de la FAQ. En effet, alors que la FAQ rapatrie tous ses services et devient autonome, elle développe d'autres programmes. À partir de ce développement, son offre se transforme : plusieurs agriculteurs qui offraient le Gîte à la Ferme préfèrent maintenant offrir la formule du Gîte du Passant, celle-ci étant peu exigeante sur le plan de l'animation. Grâce au fait que ce programme d'hébergement n'est plus seulement offert par des agriculteurs et grâce à la création du programme Auberge du Passant (en 1992), la FAQ vit un développement important, tant en milieu rural et agricole qu'urbain. Ajoutons que la création du programme Auberge du Passant dans les années quatre-vingt-dix a aussi contribué à ce développement.

Aujourd'hui, seulement $4 \%$ des membres de la FAQ n'offrent pas d'hébergement mais seulement $6 \%$ des membres offrent au moins un des programmes d'agrotourisme lié à l'animation ou à la restauration à la ferme. Ce constat semble plutôt contradictoire, alors que le Québec connaît depuis quelques années un véritable engouement pour l'agrotourisme, particulièrement sur le plan gastronomique (restauration à la ferme, produits de la ferme, produits des terroirs régionaux, etc.).

Consciente de ces enjeux, la FAQ a entrepris au cours des dernières années une vaste réflexion sur la situation de l'agrotourisme au Québec. Au terme de cette démarche, elle a élaboré un plan de développement qui comporte un ensemble de mesures pour relancer l'ensemble de son offre agrotouristique. Le projet s'inscrit dans la perspective de la conjugaison des efforts des intervenants et selon les orientations du Groupe de Concertation en Agrotou- risme. Sur la base d'un partenariat avec ce Groupe, la FAQ veut réaliser ce projet de développement.

\section{La tournée provinciale}

À la suite de cette prise de conscience, la FAQ a décidé de donner un nouveau souffle aux programmes de restauration et d'animation du volet agrotouristique au sein de son organisation. Après une réorganisation de ses ressources humaines, en juin 2000, en février-mars 2001, une grande tournée provinciale a été instiguée avec l'aide des membres du Comité agrotouristique de la FAQ et de l'UPA. Tous les producteurs engagés dans l'agrotourisme et les intervenants qui travaillent à son développement ont été conviés à un forum dans chacune des régions du Québec. Les objectifs de la tournée étaient :

- de faire connaître la FAQ aux producteurs et aux intervenants ;

- de sensibiliser les producteurs aux avantages du réseau ;

- de leur faire connaître la situation de l'agrotourisme dans leur propre région ;

- d'entreprendre une réflexion sur les enjeux de développement ;

- d'identifier des projets collectifs pour appuyer les entreprises et les circuits agrotouristiques.

Le bilan de cette grande tournée a ainsi donné à la FAQ ses grandes lignes d'action en matière d'agrotourisme. Celles-ci concernent :

- la définition de l'agrotourisme,

- la reconnaissance du secteur,

- le positionnement de la FAQ, 
- les critères de qualité,

- la réglementation sur la qualité des aliments,

- l'aménagement et la protection du territoire agricole,

- la mise en marché de l'agrotourisme.

Dans cette optique, l'hypothèse de l'intégration des circuits agrotouristiques à l'offre de la FAQ a été émise. Un projet pilote permettant de structurer le développement des circuits est envisagé.

\section{L'étude sur la caractérisation des circuits agrotouristiques au Québec}

Directement tirée des commentaires recueillis, cette dernière recommandation se trouve à la base de l'étude sur la caractérisation des circuits agrotouristiques menée au sein de la FAQ. Outre un intérêt général pour le secteur agrotouristique, les objectifs de l'étude ont une portée d'intérêt particulier pour l'organisation des circuits :

- dresser un portrait qualitatif et quantitatif des circuits agrotouristiques au Québec et dans les régions,

- faire la caractérisation des circuits agrotouristiques,

- identifier et analyser les problématiques de développement,

- proposer des moyens de structurer le développement des circuits et de favoriser leur réseautage.

À long terme, l'étude vise à valider l'hypothèse selon laquelle la création d'un programme de circuits agrotouristiques à la FAQ pourrait impulser la structuration du travail en réseau de l'agrotourisme au Québec.

La démarche méthodologique suivie a commencé avec la rédaction d'un questionnaire qui visait à obtenir de l'information générale sur le contexte agrotouristique de chaque région ainsi que de l'information sur les circuits répertoriés, mais aussi à sonder l'opinion et le degré d'adhésion des intervenants au réseautage; le tout afin de dégager un portrait de circuit généralisable.

Le questionnaire se divise en sept parties. La première partie porte sur le contexte agrotouristique de la région, c'est-à-dire le potentiel au sein de l'offre touristique, ses forces et ses faiblesses. Les autres étapes concernent uniquement le contenu des circuits. Elles permettent de comprendre d'où vient l'initiative du circuit et la démarche suivie, notamment 1'origine institutionnelle et les caractéristiques du circuit. La suite des questions porte sur les activités menées, les opérations de commercialisation, les outils de promotion et les difficultés rencontrées dans le but d'évaluer le dynamisme et la vie du circuit. Enfin, nous avons cherché à connaître la structure fonctionnelle du circuit ( $\mathrm{co}$ mité organisateur, cahier des charges, processus de contrôle de la qualité...). En résumé, nous avons cherché à connaître tout ce qui entoure la gestion des circuits agrotouristiques.

L'étape de la réalisation complétée, il fallait passer à l'administration du questionnaire. Dans un premier temps, nous avons identifié les régions concernées par l'agrotourisme (il y en a quinze). Dans ces régions, les responsables, employés ou initiateurs des circuits ont été contactés pour connaître leur degré de coopération à l'étude. Les contacts ont été pris au sein des Centres locaux de développement (CLD), des Tables de concertation, des bureaux de l'UPA ou du MAPAQ, des Offices de Tourisme et des Associations touristiques régionales (ATR). Souvent, les personnes contactées ont identifié d'autres intervenants susceptibles de nous renseigner.

Finalement, vingt-quatre personnes ont reçu le questionnaire par courrier électronique. Un suivi a été fait pour valider le questionnaire et répondre aux questions les plus immédiates. Au total, douze questionnaires ont été retournés dans un délai d'environ trois semaines, soit un taux de réponses de $50 \%$.

\section{Portrait de l'offre agrotouristique et des circuits au Québec et dans les régions}

Au total, 1059 exploitations agricoles mènent des activités du domaine de l'agrotourisme ou dans un domaine connexe (tableau 1). Ces activités représentent
$3,35 \%$ du total des agriculteurs qui est de 31595 au Québec (MAPAQ, 2001b).

La majorité de ces activités, soit $74 \%$, se rapporte à la vente de produits (près de $60 \%$ de cette catégorie), à l'auto-cueillette (31\%), puis à la dégustation ainsi qu'à la chasse et à la pêche $(9 \%)$. Ces activités constituent la première étape de l'ouverture d'une exploitation au public. Elles sont simples à développer - moins élaborées que les visites guidées ou l'hébergement - et sont basées sur les produits de la ferme.

Ensuite, $45 \%$ des agriculteurs développant des activités touristiques organisent des visites et des animations, principalement des visites de ferme (visites guidées ou visites éducatives). Le reste de la catégorie concerne les balades, les randonnées, les centres d'interprétation et l'observation, dans de faibles pourcentages. Dans de plus faibles proportions, nous trouvons les expositions, les musées et les documentaires vidéos.

La restauration représente $25 \%$ des activités agrotouristiques. Les aires de piquenique, les repas champêtres et les repas de cabanes à sucre représentent chacun $10 \%$ environ du total des activités. Les méchouis sont une autre forme peu développée.

Enfin, $6 \%$ des activités se rapportent à l'hébergement. Dans cette catégorie, les Gîtes du Passant représentent la majorité de l'offre, suivis, dans l'ordre, des hébergements divers, des Auberges du Passant et des Gîtes à la Ferme.

En ce qui concerne le portrait des circuits, le tableau 1 illustre la variété des activités agrotouristiques proposées. Cela est en fait très favorable à la constitution de circuits. Nous avons une diversité qui à la fois représente le patrimoine agricole québécois et varie l'offre proposée aux touristes. Il reste à savoir si ces activités sont de qualité et si elles sont commercialisables.

D'une manière générale, la région des Laurentides est la région qui regroupe le plus grand nombre d'exploitants agricoles pratiquant des activités agrotou- 


\begin{tabular}{|c|c|c|}
\hline \multicolumn{3}{|c|}{ 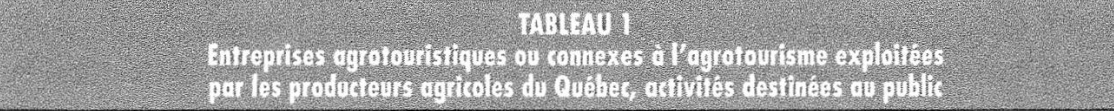 } \\
\hline Activités destinées au public & Nombre & En $\%$ du fotal \\
\hline Visite ef animation & 473 & $45 \%$ \\
\hline Restauration & 261 & $25 \%$ \\
\hline Hébergement & 60 & $6 \%$ \\
\hline Promotion et vente de produits & 780 & $74 \%$ \\
\hline Entreprises agrotouristiques ou connexes à l'agrotourisme & 1059 & $100 \%$ \\
\hline
\end{tabular}

Source: $M A P A Q, 2001 a$

ristiques, loin devant la Montérégie, Chaudière-Appalaches, le Centre du Québec, Laval, le Saguenay-Lac-St-Jean et Lanaudière (MAPAQ, 2001a). Cependant, la région de Laval possède un potentiel proportionnellement plus important que les autres régions : en effet, près de $50 \%$ de ses exploitants agricoles mènent des activités agrotouristiques. Nous remarquons aussi qu'un producteur engagé dans l'agrotourisme l'est rarement pour une seule et unique activité. En général, il mènera plus d'une activité.

\section{Analyse des résultats}

\section{État de la situation}

Agrotourisme ou tourisme rural ? En dépit du fait que le Groupe de concertation sur l'agrotourisme a adopté une définition de l'agrotourisme, force est de constater qu'elle n'est pas mise en application par l'ensemble des intervenants ${ }^{1}$. En fait, il règne encore une relative confusion entre les termes utilisés lorsqu'il s'agit de faire la promotion des produits agroalimentaires, 1'agrotourisme et le tourisme rural. Même si la plupart des intervenants souscrit à la définition, son application ne se fait pas sans difficultés. À titre d'exemple, quand un organisme veut publier un outil de promotion ou encore mettre sur pied un circuit agrotouristique, il a tendance, pour financer le projet, à recruter le plus d'entreprises possibles sans qu'elles répondent nécessairement à la définition.
La plupart des circuits agrotouristiques recensés font davantage référence à une route touristique dans laquelle on trouve une variété d'attraits. De l'avis de certains intervenants touristiques, l'agrotourisme semble mieux « se vendre » que le tourisme rural, mais il est plus difficile à mettre en place.

Notons que sur les douze répondants au sondage des circuits, huit sont des circuits de tourisme rural, soit $67 \%$, trois sont thématiques et un seul est purement agrotouristique.

\section{Les forces et les faiblesses de l'agrotourisme}

D'une manière globale, en ce qui concerne les activités agrotouristiques, la situation est très semblable dans l'ensemble du Québec avec une offre agrotouristique forces et les faiblesses observées en région sont sensiblement les mêmes, sauf quelques différences dues à des spécificités géographiques, territoriales (proximité des villes) ou des attraits touristiques. Cependant, toutes les régions sont conscientes de la valeur de leur terroir régional et toutes mettent en œuvre sa valorisation, chacune dans ses spécificités. l'agrotourisme qui permettront un travail en réseau sont les suivants : la diversité agricole et paysagère, les efforts réalisés complémentaire et en développement. Les

Dans l'esprit de l'étude, les avantages de pour la qualité des infrastructures d'accueil, la prise de conscience des intervenants agricoles et touristiques régionaux, notamment grâce à la mise en place d'un partenariat et à une participation accrue. L'agrotourisme est, pour toutes les régions, un potentiel à développer.

Les contraintes de l'agrotourisme sont : le petit nombre d'entreprises, leur dispersion et leur diversité, la faiblesse du réseau et de l'engagement des agriculteurs, un secteur mal structuré, des infrastructures d'accueil restant à développer, un dédoublement des efforts de promotion dans les Municipalités régionales de comtés (MRC) ou les municipalités, un manque de professionnalisme, d'expertise touristique et trop de sollicitations financières de toutes parts pour les exploitants.

\section{Le processus de création d'un circuit}

Les acteurs de la mise en place d'un circuit sont de diverses natures et origines professionnelles. La grande majorité des circuits sont nés sur l'initiative de gens du milieu, de producteurs ou d'agents de développement. Trois types d'acteurs sont identifiables. Deux ou trois circuits ont su combiner des forces différentes et complémentaires : une personne du milieu touristique, un producteur et un intervenant agricole. Très souvent, il s'agit d'employés de structures institutionnelles ou gouvernementales; ils dépendent soit de l'agriculture soit du tourisme. Ils appartiennent au MAPAQ, à l'UPA, aux CLD ou aux autres structures associées à l'agroalimentaire. D'autres sont des employés de Tourisme Québec, des Associations touristiques régionales, des Offices de Tourisme ou des volets tourisme des MRC. Dans certaines régions, l'initiative est principalement supportée par des agriculteurs bénévoles, comme dans 1'Outaouais ou Charlevoix. Il s'agit de personnes très engagées dans la vie de leur région et possédant de fortes convictions quant à l'activité agrotouristique. Le dynamisme dont ces personnes font preuve peut vite être absorbé par un manque de disponibilité. De plus, face au manque d'intérêt des autres forces locales, il arrive que ces agriculteurs engagés se découragent. 
Avec l'initiative des circuits, l'objectif a été en général de développer et de valoriser l'activité agricole en se concertant et en offrant une activité complémentaire. De cette manière, on trouve le moyen de faire connaître les productions, les produits et les fermes d'une région. Il s'agit aussi de construire un lien entre le monde agricole et les visiteurs et de valoriser le patrimoine local. Pour tous ces circuits, l'objectif principal est le soutien au secteur, la structuration et le développement de l'offre, sa promotion et sa commercialisation.

Comment sont nés ces circuits et quelles actions ont-ils menées? Les démarches suivies par les acteurs sont variées. Nous distinguons trois cas :

- Un des circuits est parti d'une étude de la demande. Il est implanté dans une région à proximité d'un centre urbain important. En effet, les intervenants ont observé qu'il existe une fréquentation de la clientèle urbaine qui est intéressée par les activités récréatives et éducatives liées au monde rural ou agricole. L'achalandage se trouve à proximité. Nous sommes donc en présence d'une démarche « marketing ».

- Un autre circuit s'est bâti grâce à l'étalonnage concurrentiel (benchmarking), c'est-à-dire grâce à l'observation de la concurrence. Les intervenants se sont inspirés de ce qui se faisait ailleurs en agrotourisme en visitant des sites agrotouristiques au Québec et ailleurs.

- Les autres circuits, la majorité, se sont attachés à l'offre sur leur territoire. Sur une même zone, il existe un potentiel agrotouristique et une vie rurale active, mais peu valorisée. Il s'agit dans ce cas d'un soutien et d'une mise en valeur du potentiel local qui rejoignent directement les objectifs d'un réseau, soit le développement et la valorisation de l'activité agricole, de ses acteurs et de ses productions, la création de liens entre le monde agricole et les visiteurs, mais aussi la valorisation générale du patrimoine rural local.

La démarche pour construire un circuit commence par le regroupement et la concertation de tous les acteurs potentiels et intéressés par l'idée. Des outils de promo- tion sont réalisés, puis vient l'animation du réseau avec des conférences, des réunions et des formations.

Pour certains circuits, l'évolution de la situation est très instable. Alors que certains sont en développement constant, d'autres ne sont pas reconduits d'une année à l'autre. Les actions menées s'établissent soit à long terme, comme la promotion, la formation, le développement des produits ; soit ponctuellement avec des évènements ou des fêtes estivales.

\section{La commercialisation et la promotion}

La promotion constitue un volet bien maîtrisé par les organisations qui gèrent les circuits. Elles mènent toutes des actions promotionnelles classiques : cartes, dépliants, guides, site Internet, signalisation. La diffusion reste régionale ou dans les zones urbaines proches (Ottawa, Montréal ou Québec, selon la région). Elles ont recours aux salons touristiques et aux médias grâce à des tournées médiatiques ou à des articles. De plus, les subventions versées par le gouvernement sont destinées à la promotion. Cependant, aucune organisation ne peut mesurer les retombées de ces efforts promotionnels. Ces organisations donnent une estimation générale et approximative basée sur l'observation empirique de faits : les entreprises semblent satisfaites, l'Office de Tourisme leur a affirmé que les touristes prenaient leur dépliant, etc. Les intervenants jugent soit que leurs efforts sont très bons, soit qu'ils sont médiocres.

Ce constat soulève le problème de la multiplication des coûts. S'il veut bénéficier du soutien promotionnel offert par les différents organismes (CLD, ATR, FAQ, etc.), le propriétaire d'entreprise agroutouristique doit payer plusieurs cotisations, ce qui représente dans certains cas un montant important. Il s'agit d'un irritant majeur pour les promoteurs, d'autant plus que les retombées sont parfois incertaines. Dans les faits, il existe une concurrence entre les organismes pour obtenir une part du budget du producteur qui est destiné à la promotion. L'inscription dans plusieurs guides touristiques est certes bénéfique pour l'entreprise, dans la mesure où l'on positionne les produits agrotouristiques sur les différents marchés. À ce chapitre, il y a lieu de se questionner sur l'efficacité de certains outils utilisés pour promouvoir l'agrotourisme. De plus, rares sont ceux qui ont recours à un plan de marketing pour positionner l'agrotourisme et ses produits dérivés dans une perspective à moyen et à long terme. Pour être en mesure de faire des choix plus éclairés, les producteurs aimeraient connaître les coûts et les bénéfices liés au soutien offert par les différents réseaux.

En ce qui concerne la commercialisation, des efforts restent à faire. La situation est très hétérogène et seulement cinq organisations interrogées sur les douze (40\%) ont un plan de commercialisation pour leurs circuits. Quant aux accords de commercialisation avec des agences de voyages, aucune norme n'existe. Ces projets se sont souvent soldés par des échecs à cause d'un manque de rentabilité observé par les agents de voyages. Il semblerait pourtant que certains circuits s'orientent vers des accords avec les ATR qui sont de plus en plus intéressées à l'agrotourisme.

\section{Le processus d'accréditation et de contrôle de la qualité}

Seulement trois organisations gérant des circuits agrotouristiques sur les douze organisations interrogées possèdent un cahier des charges pour ces circuits. Leur contenu reste pourtant sommaire. En effet, ces circuits s'appuient sur des critères non applicables aux autres circuits. Cela relève d'un caractère très social. La majorité des organisations interrogées estime qu'il ne s'agit pas d'une étape primordiale étant donné que les responsables des circuits, les membres et les comités d'administration le cas échéant, ont la capacité de juger les membres du circuit. Pour les circuits gérés par des agriculteurs bénévoles, l'absence d'un cahier des charges est davantage due à un manque de temps et de moyens pour le réaliser.

De même, les méthodes de sélection, d'accréditation et de contrôle de qualité 
existent, mais elles sont souvent informelles et reposent sur une évaluation subjective. Par exemple, le producteur est connu depuis longtemps, il peut être membre et accueillir des visiteurs. Six circuits n'ont pas de processus d'accréditation et de contrôle qualité ; cinq en possèdent un, informel, et un seul s'est doté d'un processus formel avec un inspecteur.

Il est important de ne pas effacer l'aspect humain et relationnel, l'inter-connaissance ; mais un cahier des charges avec des critères stricts et objectifs constitue une base solide. Ce point est forcément à prendre très au sérieux si l'on veut un programme à part entière, crédible auprès de la clientèle et reconnu par les professionnels.

\section{La structure organisationnelle des circuits agrotouristiques}

La moitié des circuits sondés possèdent un statut informel, sans comité d'administration. Quatre ont le statut d'organisme à but non lucratif et deux relèvent d'organisations formelles. Ces six derniers se sont dotés d'un comité formel - constitué d'intervenants et de membres des circuits - qui supervise le circuit, l'oriente et prend les décisions. De plus, il ne s'agit pas toujours de structures réellement autonomes. Bien souvent, ces circuits sont gérés parmi d'autres dossiers par des réseaux ou des conseils agricoles, bio-alimentaires ou agroalimentaires locaux.

En général, tous les organismes concernés ont une personne ressource très engagée, souvent initiatrice du circuit, qui joue le rôle de coordonnateur. Ces personnes portent beaucoup de responsabilités, ce qui ne s'avère pas toujours favorable ni pour l'intérêt général ni pour l'intérêt des personnes concernées. Il ne s'agit pas forcément d'une méthode très démocratique, étant donné que les réunions de collaboration ou les tables de discussions entre décideurs et membres se font rares.

\section{Les ressources humaines et financières}

Les ressources humaines sont prêtées par les organismes précédemment cités (avec un employé ou, rarement, plusieurs em- ployés). Ils portent généralement le titre d'agent de développement et consacrent une partie de leur temps au dossier, parmi d'autres dossiers. Ce ne sont pas des postes spéciaux ou des personnes détachées spécialement, mais ces personnes sont toujours secondées - quand elles ne sont pas totalement suppléées - par des bénévoles. Sur certains circuits, des agriculteurs ou d'autres acteurs agissent de manière volontaire et bénévole, gérant la majorité du circuit.

Les budgets dont disposent les circuits varient fortement selon les cas, de $2200 \$$ à $100500 \$$ pour deux années. Quand on constate ces écarts on comprend facilement les inégalités entre circuits.

\section{Les services offerts aux membres}

Les services offerts par les organismes qui supportent les circuits touchent essentiellement la promotion, la commercialisation des produits et les activités des entreprises agrotouristiques. L'objectif de ces appuis est d'accroître les retombées pour les entreprises et les collectivités. La formation des membres au volet touristique de leur entreprise (comptabilité, aménagements, investissements...) est souvent proposée. N'oublions pas que la qualité et la fréquence de ces initiatives de formation varieront avec les moyens humains et financiers du circuit. Les résultats de notre enquête démontrent qu'un seul circuit ne propose aucun service à ses membres.

\section{Le financement des circuits}

Pour sept circuits sur les douze, les sources de financement sont à la fois internes et externes. Le financement interne comprend les cotisations annuelles des membres et le budget de la structure (c'est-à-dire des organisations participant aux circuits, notamment les MRC et le CLD). Le financement externe regroupe les subventions du MAPAQ combinées aux aides de commanditaires privés. Deux circuits se financent grâce à des ressources internes et aux subventions du MAPAQ. Les trois autres circuits sont financés soit par des ressources externes uniquement, soit par des ressources internes uniquement, soit par des ressources internes et des commandites.
Nous avons observé précédemment que les budgets varient fortement. Les acteurs doivent s'y adapter et c'est là où le bât blesse. Face aux producteurs, les responsables des circuits ne peuvent pas se permettre d'instaurer des cotisations trop élevées car, ajoutés à d'autres adhésions, les coûts se multiplient. Mais ils éprouvent aussi de la difficulté à obtenir des aides régulières et suffisantes du gouvernement. Bien souvent, ils n'ont pas assez de ressources pour financer un poste à plein temps sur le dossier circuit.

\section{Quelles orientations stratégiques adopter en faveur de l'agrotourisme?}

\section{Coûts et disponibilité}

Les problèmes soulevés par les circuits ainsi que par une mise en réseau sont la disponibilité des acteurs ainsi que les coûts à débourser pour les exploitants et les sources de financement en général. Nous parlons ici du réseau agrotouristique provincial qui serait constitué sur la base des circuits existants grâce au travail coordonné de la FAQ ainsi que des divers intervenants et institutions impliqués. Le réseau agrotouristique provincial se positionnerait alors comme une structure unique concentrant les budgets alloués par les partenaires et fournissant des moyens humains spécialisés, strictement mandatés au dossier réseautage agrotouristique. Il tiendrait le rôle de coordonnateur à l'échelle provinciale en dépêchant les aides humaines et financières dans les régions.

\section{Un réseau pour structurer}

Il n'existe pas de structure régionale de la FAQ qui - à l'image des Relais Départementaux des Gîtes de France - pourrait aider les agriculteurs et accroître leur participation. Une animation du réseau reste à organiser. Elle passera par 1'information et la formation des exploitants, la promotion de l'agrotourisme régional avec des regroupements en réseau, des petites activités concrètes et ponctuelles pour que les membres se connaissent mieux. 


\section{Un réseau pour soutenir}

Agriculteur, transformateur ou restaurateur sont des professions très spécialisées ne pouvant pas posséder toutes les subtilités de l'industrie touristique. Elles bénéficient d'une grande expertise dans leur domaine et relèvent de la vocation car elles demandent énormément d'investissements financiers et humains. Dans un réseau, ces acteurs ont besoin de trouver un réel soutien et une coordination qu'ils ne peuvent acquérir seuls. Le réseau et ses personnesressources devront conseiller, animer, former, regrouper, réfléchir à la promotion et à la commercialisation. Cela constituerait, pour eux, une approche globale.

\section{Un réseau pour la qualité et le caractère local}

Se regrouper pour travailler ensemble, regrouper les circuits, établir des normes, ce n'est pas standardiser. Il ne faut pas imposer un carcan, mais laisser les forces locales libres d'imagination en les aidant et en conservant les couleurs locales, sans omettre le rôle des intervenants. Le réseau doit intervenir pour assurer la qualité demandée par les touristes et la professionnalisation du milieu.

\section{La demande et la proximité de I'urbain}

Concernant la demande, il ne faut pas oublier que l'offre a un meilleur achalandage si elle se situe à proximité d'un foyer urbain, car le tourisme rural a une portée régionale et urbaine. De plus, il est important de prendre en compte les attentes de la clientèle individuelle : elle ne veut pas d'un long séjour à thème agrotouristique pur. Elle veut de courts séjours qu'elle peut constituer à la carte avec plusieurs activités de différentes natures : culturelles, sportives, etc.

\section{Les attentes vis-à-vis de la FAQ}

Grâce aux sondages sur les circuits, nous mettons le doigt sur de nouveaux constats, inconnus de la FAQ. Ce sont des points intéressants, moins critiques et plus concrets que ceux recueillis lors de la tournée provinciale. Le rôle de la FAQ devra s'orienter vers ces cinq axes :

- Acquérir un réel rôle d'acteur agrotou= ristique ;

- Conserver son rôle de professionnel du tourisme ;

- Se charger de l'accréditation et du contrôle qualité des circuits ;

- Porter la promotion et l'animation des circuits ;

- S'engager sur le terrain, avoir un rôle concret et constructif.

Vis-à-vis de la FAQ, nous notons une réelle attente d'un rôle pragmatique et fédérateur. La FAQ pourrait prendre le rôle d'un « syndicat agrotouristique », comme un organe d'appui aux exploitants, de conseil sur le terrain. Son activité ne serait pas seulement promotionnelle, mais surtout constructive pour les entreprises et respectueuse de la réalité agrotouristique locale. Les sondés s'attendent à ce qu'elle acquiert une attitude très engagée sur le terrain dans le vrai sens de «fédération».

\section{Conclusion}

L'agrotourisme constitue encore une offre complémentaire dans l'offre touristique provinciale et régionale au Québec. Il acquiert peu à peu ses galons et se bâtit.

Devant ces constats, il reste, concrètement, à mettre en place un réseautage. En s'appuyant sur le programme de circuits à l'échelle du Québec, le projet de développement parviendra certainement à constituer un réseau et à donner une place à l'agrotourisme.

La FAQ doit intégrer les circuits dans son offre avec l'objectif de la mise en réseau et de l'augmentation de l'engagement des membres dans ce réseau. Elle a le devoir d'aider les acteurs de terrain et de se présenter comme un vrai moyen de mise en réseau. Les intervenants comme les producteurs attendent des mesures palpables; ils sont prêts à se mettre à l' œuvre. La FAQ constitue elle-même l'un des moyens concrets à l'initiation du réseau. Le secteur estil prêt à prendre ce tournant ?
Ne l'oublions pas : la FAQ est avant tout un réseau humain qui fusionne le dynamisme et l'énergie de ses membres au travail de ses permanents. C'est sur eux que nous pouvons compter pour développer l'agrotourisme structuré et concerté.

Anne Marchard termine sa quatrième année d'études supérieures de Tourisme et d'Hôtellerie à l'Université d'Angers (France) ; elle a été stagiaire à la Fédération des Agricotours du Québec pour le volet agrotourisme, du mois de mars au mois de juin 2001.

\section{Note}

1 L'agrotourisme est alors défini comme une « activité complémentaire à l'agriculture. Il met en relation des producteurs et productrices agricoles, avec des touristes ou des excursionnistes, permettant ainsi à ces derniers de découvrir le milieu agricole, l'agriculture et sa production à travers l'accueil ainsi que la communication des savoirs et des savoir-faire que leur réserve leurs hôtes. Afin de préserver son caractère d'authenticité et sa crédibilité, l'agrotourisme se doit d'être centré sur les producteurs et productrices et les productions agricoles. Tout circuit agrotouristique doit reposer à la base sur des exploitations agricoles », (Groupe de Concertation sur l'agrotourisme, 2001).

\section{Bibliographie}

Groupe de Concertation sur l'agrotourisme (2001), Problématiques de développement de l'agrotourisme en regard à l'aménagement et à la protection du territoire agricole, Groupe de Concertation sur l'agrotourisme et du Comité sur l'aménagement et la protection du territoire agricole.

Ministère de l'Agriculture, des Pêcheries et de l'Alimentation du Québec (2001), http:// www.agr.gouv.qc.ca.

Ministère de l'Agriculture, des Pêcheries et de l'Alimentation du Québec (2001a), Entreprises agrotouristiques ou connexes à l'agrotourisme exploitées par les producteurs agricoles du Québec, activités destinées au public, Banque de données du Groupe de concertation sur l'agrotourisme au Québec, 1 p.

Union des Producteurs Agricoles (1999), Document d'orientation sur l'agrotourisme, Montréal, $157 \mathrm{p}$. 